\title{
Increased dose of re-irradiation therapy improves the survival of patients with local recurrent esophageal squamous cell carcinoma after radiotherapy
}

\section{Xun Wu ( $\nabla$ yjs2016nsmc@sina.com)}

Chengdu Fifth People's Hospital

\section{Xingsheng Hu}

The Second Xiangya Hospital of Central South University

Junru Chen

Sichuan Cancer Hospital and Research Institute

\section{Lang He}

Chengdu Fifth People's Hospital

\section{Research}

Keywords: esophageal squamous cell carcinoma, recurrence, salvage chemoradiation therapy, salvage radiation therapy

Posted Date: December 12th, 2020

DOI: https://doi.org/10.21203/rs.3.rs-56036/v2

License: (c) (1) This work is licensed under a Creative Commons Attribution 4.0 International License.

Read Full License 


\section{Abstract}

Introduction: Local recurrence (LR) threatens the treatment of esophageal squamous cell carcinoma (ESCC). This study interrogated the optimal re-irradiation dose for the LRESCC following radical (chemo) radiotherapy.

Methods: We retrospectively analyzed a total of 125 patients with LRESCC after initial radiotherapy. For the radiotherapy dose, 58 patients were assigned to low-dose (LD) group (50-54 Gy) while the remaining 67 were classified into the high-dose (HD) group (55-60 Gy). We recorded the response rate (complete + partial response), 1-, 2- and 3-year survival rate, and toxicity. We then analyzed the impact of the different radiotherapy doses, and combination chemotherapy on the survival of the LRESCC patients.

Results: After re-irradiation, the 1-, 2- and 3-year survival rates were $48.3 \%, 24.1 \%$ and $10.3 \%$ in the LD group, and $61.2 \%, 34.3 \%$ and $19.4 \%$ in the HD group $(P<0.05)$, respectively. The median survival time of patients receiving radiotherapy alone was 9 months in the LD group and 15 months in the HD group $(P<0.05)$. Whereas the survival rate of patients treated with chemoradiotherapy was higher than that of patients treated with radiotherapy alone in the LD group, chemoradiotherapy showed no advantage over radiotherapy alone in the HD group. In addition, the incidence of esophagitis, the most common toxicity, was higher in the HD group compared to the LD group ( $68.7 \%$ vs $58.6 \%, P<0.05)$. Our multivariate analysis demonstrated that re-irradiation dose was an independent favorable prognostic factor in patients with LRESCC.

Conclusion: Taken together, our data shows that, increasing the re-irradiation therapy dose (55-60Gy) improves the long-term survival of patients with LRESCC after radiotherapy, with tolerable toxicity.

\section{Introduction}

Esophageal squamous cell carcinoma (ESCC) is the most common esophageal malignancy in China. Radiotherapy and surgery are the mainstay treatment option for the ESCC. Unfortunately, the local recurrence rate of esophageal cancer remains at $40 \%-60 \%$, following radiotherapy [1]. Without active intervention, most of the patients with disease progression die within 1 year $[2,3]$. To date, there is still no consensus on the retreatment of LRESCC following (chemo) radiotherapy. Previous studies have shown that salvage surgery is effective in the treatment of LRESCC with radiation history [4-8]. However, fibrosis of tissues surrounding the tumor bed after radiotherapy complicates the surgical intervention and increases perioperative risk, even with carefully selected patients $[9,10]$. Besides, Chen et al. reported that re-irradiation can achieve similar survival outcome as salvage surgery [2]. Therefore, re-irradiotherapy remains an important salvage treatment option for patients with LRESCC $[2,11]$. Radiotherapy dose is a vital factor that influence survival outcome [12]. Studies have shown that a salvage radiation dose $>50$ Gy and < 60 Gy yields better survival for LRESCC $[13,14]$. However, there is lack of literature on whether higher doses of re-irradiation, between 50 and $60 \mathrm{~Gy}$, would be more beneficial to patients with LRESCC 
after initial radiation. Here, we interrogated the survival outcome and toxicity of different re-irradiation doses combined with or without chemotherapy in the treatment of LRESCC.

\section{Patients And Methods}

\section{Patients and clinical features}

We retrospectively selected 207 patients with LRESCC who underwent re-irradiation therapy in the Chengdu Fifth People's Hospital between January 2012 and December 2016. We included patients with a non-recurrence interval of $>6$ months after initial radiotherapy, confirmed (pathological examination, imaging or gastroscopy) recurrence of primary esophageal cancer with or without local lymph node metastasis, or only local lymph node metastasis (supraclavicular fossa, mediastinal, esophageal, or paraaortic lymph nodes), re-irradiation therapy dose 50-60 Gy, as well as those with the Eastern Cooperative Oncology Group (ECOG) score 0-2, with functional heart, liver, kidneys, lungs, and bone marrow hematopoiesis. We excluded patients with distant organ metastasis, tumor in other organs, or those with incomplete information. Patients with hyper-fractionated radiotherapy and a history of surgical resection of esophageal cancer were also excluded. Finally, 125 patients were included, 84 men and 41 women with a median age of 68 years (range 50-89 years). All the patients had either refused surgical intervention or were unable to undergo salvage surgery. The locally recurring tumors of all the patients were located in the previously irradiated area. This study was approved by the Ethics Committee of Chengdu Fifth People's Hospital.

\section{Treatment and follow-up}

Two-dimensional radiotherapy, three-dimensional conformal radiotherapy (3D-CRT) or intensity modulated radiotherapy (IMRT) was used for the initial radical treatment for the 125 patients, with a median dose of $60 \mathrm{~Gy}$ (50-66 Gy). Most of the patients received chemotherapy regimens containing cisplatin, paclitaxel or fluorouracil. After the initial treatment, gastroscopy, esophageal barium meal radiography, or enhanced computed tomography (CT) was used to evaluate the efficacy. All the patients achieved either complete or partial response.

All the patients received re-irradiation with 3D-CRT or IMRT of 1.8-2.0 Gy per fraction, 5 days/week. Gross tumor volume (GTV) was assessed by esophageal barium meal examination and CT images. GTV for the metastatic lymph nodes (GTVnd) included enlarged lymph nodes with a short diameter $>10 \mathrm{~mm}$, or positron emission tomography-CT examination suggested metastatic lymph nodes. Planning target volume (PTV) was formed by extending $0.5-1.0 \mathrm{~cm}$ radially from the GTV, and $1.0-2.0 \mathrm{~cm}$ above and below the GTV, or $0.5 \mathrm{~cm}$ outside the GTVnd in all directions. The lymphatic drainage area was not prophylactically irradiated. Dose limitation for normal tissues or organs was: bilateral lungs $\mathrm{V} 20<25 \%$, spinal cord Dmax $<20 \mathrm{~Gy}$, and mean radiation dose to the heart $<30 \mathrm{~Gy}$. 
The overall survival (OS) was defined as time from the beginning of retreatment until last follow-up or patient death. Efficacy was evaluated at the end of retreatment and 1 month after the end of retreatment. Patient evaluation methods included endoscopy, tumor markers, esophageal barium meal, or enhanced CT. The response rate (RR) was defined as the percentage of patients with complete response and partial response in the total number of cases. Therapeutic evaluation criteria referred to response evaluation criteria in solid tumors (RECIST 1.1). In addition, toxicity was graded by the Common Terminology Criteria for Adverse Events (CTCAE v4.0). According to the re-irradiation dose, 58 patients were assigned to the low-dose (LD) group (50-54 Gy, median dose $50 \mathrm{~Gy}$ ) while the remaining 67 to the high-dose (HD) group (55-60Gy, median dose 60Gy).

\section{Statistical analysis}

We employed SPSS version 23.0 (IBM Corporation, Armonk, NY, USA) to analyse the data in this study. The continuous or categorical variables in the two treatment groups were compared by the Student's $t$ test or the chi-square test, respectively. The survival rates were calculated by Kaplan-Meier method, and the difference in survival curves was compared by log-rank method. Cox's proportional hazards regression model was used to determine the effect of univariate or multiple factors on the survival. Time to recurrence (TR) was defined as the time between the beginning of initial treatment and confirmed recurrence. Two-sided $P<0.05$ was considered to be statistically significant.

\section{Results}

The clinical baseline characteristics for the two patient groups are as shown in Table 1. Sixty-eight (54.4\%) patients relapsed within 2 years following initial definitive radiotherapy. The median time to recurrence in all the patients was 21 months (range 8-201 months). After re-irradiation, the median follow-up time was 19 months (range 4-65 months). At the end of the follow-up, 2 patients survived in the LD group, while 7 survived in the HD group. There were 67 cases of primary recurrence (PR), 33 cases of PR with local lymph node recurrence (PR+LNR) and 25 cases of local lymph node recurrence (LNR). Sixty-one of the 125 patients received concurrent or 1-4 sequential cycles of chemotherapy, which included either single or double-drug regimens containing paclitaxel, platinum or fluorouracil. In the LD group, 8 patients received platinum-containing dual-drug sequential chemotherapy, while 17 received single-drug fluorouracil or platinum-containing dual-drug concurrent chemotherapy. On the other hand, in the HD group, 14 patients received platinum-containing dual-drug sequential chemotherapy, while 22 patients received single-drug fluorouracil or platinum-containing dual-drug concurrent chemotherapy.

\section{Treatment outcomes}

After retreatment, the RR was $87.9 \%(58 / 66)$ and $76.8 \%(43 / 56)$ in the HD and LD group respectively ( $P=$ $0.065)$. The 3 -year locoregional control rate in the LD and HD group was $3.4 \%$ and $14.9 \%$, respectively $(P$ $=0.039$ ) (Figure 1). Our data showed that the $1-, 2$ - or 3-year survival rate for the whole cohort was $56.8 \%$, 
$29.6 \%$ or $15.2 \%$ respectively, with a median survival time of 14 months. The $1-, 2$ - or 3 -year survival rate in the LD group was $48.3 \%, 24.1 \%$ or $10.3 \%$, respectively, with a median survival time of 11 months (95\% confidence interval (Cl): 8.514-13.486). On the other hand, the 1-, 2- or 3-year survival rate in the HD group was $61.2 \%, 34.3 \%$ or $19.4 \%$, respectively, with the median survival time of 18 months ( $95 \% \mathrm{Cl}$ : 12.276 23.724). Therefore, compared to the patients in the LD group, patients in the HD group showed a better survival advantage $(P=0.013)$ (Figure 2$)$.

The two groups of patients were then stratified based on their acceptance of chemotherapy. Patients who received radiotherapy alone in the HD group had a significantly better survival outcome compared to the patients who received radiotherapy alone in the LD group (median survival time: 15 months, $95 \% \mathrm{Cl}$ : 7.365-22.635 vs 9 months, $95 \% \mathrm{Cl}: 7.124-10.876, P=0.009$ ) (Figure $3 \mathrm{~A}$ ). Median survival time of patients who received chemoradiotherapy was 18 months (95\% Cl: 13.597-22.403) in the HD group, and 14 months (95\% Cl: $12.378-15.622)$ in the LD group $(P=0.490)$. (Figure $3 \mathrm{~B})$. In addition, patients in the LD group who received chemoradiotherapy showed superior median survival than patients with radiotherapy alone (14 months, $95 \% \mathrm{Cl}$ : 12.378-15.622 vs 9 months, $95 \% \mathrm{Cl}: 7.124-10.876, P=0.021)$ (Figure $4 \mathrm{~A}$ ). On the other hand, median survival time of patients with or without chemotherapy was 18 months ( $95 \% \mathrm{Cl}$ : 13.597-22.403) and 15 months (95\% Cl: 7.365-22.635) in the HD group, respectively $(P=0.947)$ (Figure 4 B).

\section{Cox regression analysis for overall sample}

We conducted a univariate and multivariate analysis to evaluate whether the dose of re-irradiotherapy affected the patient survival (Table 3). In the univariate analyses, ECOG, age, TR and re-irradiation dose were significantly associated with OS ( $P<0.05$ for each comparison). Similarly, the multivariate analysis showed that ECOG of 0-1 $(P=0.048)$, age $<70$ years $(P=0.028)$, TR $>24$ months $(P=0.001)$ and reirradiation dose of 55-60 Gy $(P=0.013)$ were independent factors for favorable OS.

\section{Toxicity}

Our toxicity analysis showed that radiation esophagitis was the most common acute toxicity in both groups. The incidence of esophagitis was $58.6 \%$ in the LD group and $68.7 \%$ in the HD group $(P=0.868)$. Other acute toxicities included hematological toxicity $(P=0.004 \llbracket$ and gastrointestinal reaction (nausea, vomiting, loss of appetite and constipation) $(P=0.732)$. Rare acute toxicity included radiation-induced pneumonitis, radiation tracheitis and skin reaction. Grade $\geq 2$ radiation-induced pneumonitis was noted in only 1 patient in the HD group. A total of 11 patients, (7 patients with chemotherapy and 4 patients without chemotherapy), developed severe treatment-related toxicity, such as esophageal perforation/fistula and bleeding. The TR of most for the 11 patients (9 / 11) was $\leq 24$ months. Within 3 months following re-irradiation, 2 cases of bleeding and 3 cases of esophageal perforation/fistula 
occurred in the LD group, while 1 case of bleeding and 2 cases of esophageal perforation/fistula occurred in the HD group. In addition, 1 case of bleeding and 2 cases of esophageal perforation/fistula occurred in the LD group, 3 months after re-irradiation. Severe late complications were observed after 6 months of reirradiation; 2 patients in each group had severe esophageal stenosis and underwent esophageal dilatation (Table 2).

\section{Discussion}

This study evaluated the survival outcome and toxicity of different re-irradiation doses combined with or without chemotherapy in the treatment of LRESCC. A recent study showed that, a radiotherapy dose of $>59.4 \mathrm{~Gy}$ after standard concurrent chemoradiotherapy (50.4 Gy) led to the achievement of complete response in patients with ESCC. The dose significantly improved local control, 5-year recurrence-free survival rate and 5-year OS rate [15]. Previously, some studies have indicated that the survival rate of LRESCC can be significantly prolonged with a $>50$ Gy dose of re-irradiation $[2,13,14]$. For instance, Kobayashi et al. demonstrated that 60 Gy was an appropriate salvage dose for LRESCC, after surgery [16]. Besides, another study pointed out that patients with LRESCC with a radiation history should be given higher doses of radiotherapy [17]. These studies suggest that increasing the dose of radiotherapy for recurrent esophageal cancer may prolong the overall patient survival.

Here, we compared the effects of 50 Gy and 60 Gy median re-irradiation dose on the survival of patients with LRESCC with radiation history. Our data showed that $54.4 \%(68 / 125)$ of the patients experienced recurrence within 2 years following initial (chemo) radiotherapy, which was consistent with previous reports $[2,13,18,19]$. In their studies, Xu et al. reported that the 2-year survival rate and median survival time of LRESCC patients receiving $\geq 50$ Gy re-radiotherapy was $37.5 \%$ and 18 months respectively [14]. Whereas this outcome was better than the whole cohort of our study, it was similar to our survival results in the HD group. In this study, the 2-year survival rate and median survival time for the whole cohort was $29.6 \%$ and 14 months respectively. The 1-,2-, or 3-year survival rate and median survival time was $61.2 \%$, $34.3 \%$ or $19.4 \%$ and 18 months in the HD group, and $48.3 \%, 24.1 \%$ or $10.3 \%$ and 11 months in the LD group, respectively. As demonstrated by our multivariate analyses, the dose of radiotherapy was an important factor in defining survival. The median survival time of patients receiving radiation alone in the HD group was 15 months, which was significantly higher than the 9 months in the LD group. Our results showed better outcome compared with previous studies $[2,13,17]$. The higher doses of radiation might have been more beneficial for tumor control. Therefore, we recommend higher doses of re-radiation for patients with recurrent ESCC after radiotherapy.

Besides, local control is a vital factor affecting patient survival outcome [20]. To date, few studies have reported the rate of local control in patients with LRESCC following re-irradiation therapy. Previous studies reported that increasing the radiation dose can significantly improve the local control rate of esophageal cancer, and survival outcome [21, 22]. Here, the 3-year locoregional control rate in the LD group and that in the HD group was $3.4 \%$ and $14.9 \%$, respectively. The outcome was discouraging probably because the lymphatic drainage was not included in the treatment field. 
Many studies have demonstrated positive effect of chemotherapy in the initial treatment of ESCC [23]. However, data on the role of chemotherapy in the re-irradiation therapy of ESCC remains very scant. Few studies have shown the role of chemotherapy in re-radiotherapy for recurrent ESCC. Chen et al. reported that the 1-, 2- or 3-year survival rate of 36 LRESCC patients receiving re-irradiation with concurrent chemotherapy (paclitaxel + cisplatin) was $51.7 \%, 21.4 \%$ or $12.2 \%$, respectively [2]. In addition, Katano et al. reported that the median survival time for 6 patients who underwent concurrent chemotherapy (nedaplatin and tegafur) with re-radiotherapy was 13.6 months [10]. In a stratified analysis, our data demonstrated that chemotherapy coupled with radiotherapy could increase survival rate. In the LD group, the median survival time for chemoradiotherapy was 14 months, which was consistent with the results reported by Katano et al. [10]. However, in the HD group, chemoradiotherapy did not show better survival benefit compared to the radiotherapy alone. Our results suggest that chemotherapy can increase the survival rate of LRESCC patients with re-radiotherapy, when patients are exposed to lower radiation doses.

Our data showed that acute radiation esophagitis was the most common toxicity in the whole cohort. Whereas the incidence of esophagitis in the HD group was higher than in the LD group (68.7\% vs $58.6 \%$ ), the difference was not significant. A previous study showed that the incidence of severe acute radiation esophagitis in patients with thoracic radiotherapy was 15-25\% [24]. Similarly, grade $\geq 3$ acute esophagitis was rare in both groups in this study. Besides, hematological toxicity and gastrointestinal reactions were also common, but only hematologic toxicity was significant between the two groups. More than two-thirds of patients with hematological toxicity or gastrointestinal reaction received chemoradiotherapy. In this study, the incidence of radiation pneumonitis was lower than that shown in previous studies [25]. In addition, grade 1-2 radiation pneumonitis occurred in six (10.3\%) and eight (11.9\%) patients in the LD and HD groups, respectively, with controllable symptoms. Severe complications occurred mainly within 3 months following re-irradiation. A total of 11 patients had severe treatment-related toxicity, such as bleeding, esophageal perforation, and esophageal fistula, eight of which were in the LD group. This may be related to the TR of $\leq 24$ months in most patients $(36 / 58$, $62.1 \%)$ in the LD group. Severe late complications; esophageal stenosis, and the dysphagia were effectively relieved after esophageal dilation. Therefore, higher re-irradiation dose may be a safe treatment option for the patients.

\section{Conclusion}

Taken together, our data demonstrates that higher re-irradiation dose (55-60 Gy) for LRESCC patients with radiation history may yield better long-term survival outcome, with tolerable toxicity. Re-irradiation with lower doses (50-54 Gy), combined with chemotherapy can improve survival outcomes of LRESCC patients with radiation history. For those patients with a short recurrence time (TR $\leq 24$ months), its important to monitor serious treatment-related toxicity.

\section{Abbreviations}


LRESCC: locally recurrent esophageal squamous cell carcinoma; LD group: low-dose group; HD group: high-dose group; ESCC: Esophageal squamous cell carcinoma; ECOG: Eastern Cooperative Oncology Group Performance Status; 3D-CRT: three-dimensional conformal radiotherapy; IMRT: intensity modulated radiotherapy; CT: computed tomography; RR: response rate ; GTV: Gross tumor volume; GTVnd: Gross tumor volume of the metastatic lymph nodes; PTV: Planning target volume; OS: overall survival; RECIST 1.1: Response Evaluation Criteria in Solid Tumors Version 1.1; CTCAE v4.0: Common Terminology Criteria for Adverse Events version 4.0; TR: time to recurrence; LNR: local lymph node recurrence; PR: primary recurrence; $\mathrm{Cl}$ : confidence interval

\section{Declarations}

\section{Ethical Approval and Consent to participate}

This study was approved by the Ethics Committee of Chengdu Fifth People's Hospital, Chengdu, China.

\section{Consent for publication}

Not applicable.

\section{Availability of data and materials}

All data generated or analyzed during this study are included in this article.

\section{Competing interests}

The authors declared that they had no competing interests.

\section{Funding}

None.

\section{Authors' contributions}

XUN WU and JUNRU CHEN participated in research design. Acquisition of the data was performed by XINGSHENG HU and XUEMEI YANG. Evaluation of the images was conducted by XINGSHENG HU and LANG HE. The manuscript written by XUN WU. All authors read and approved the final manuscript.

\section{Acknowledgements}

We thank Professor Guangming Li for his guidance on the design of this study.

\section{References}

1. Pennathur A, Gibson MK, Jobe BA, Luketich JD: Oesophageal carcinoma. The Lancet 2013, 381(9864):400-412. 
2. Chen Y, Lu Y, Wang Y, Yang H, Xia Y, Chen M, Song H, Li T, Li D, Wang J et al: Comparison of salvage chemoradiation versus salvage surgery for recurrent esophageal squamous cell carcinoma after definitive radiochemotherapy or radiotherapy alone. Diseases of the esophagus : official journal of the International Society for Diseases of the Esophagus 2014, 27(2):134-140.

3. Kim YS, Lee CG, Kim KH, Kim T, Lee J, Cho Y, Koom WS: Re-irradiation of recurrent esophageal cancer after primary definitive radiotherapy. Radiation oncology journal 2012, 30(4):182-188.

4. Harada H, Yamashita K, Katada C, Ishiyama H, Soeno T, Washio M, Sakuraya M, Ushiku H, Niihara M, Hosoda $\mathrm{K}$ et al: Patient selection for salvage surgery after definitive chemoradiotherapy in esophageal squamous cell carcinoma. Langenbecks Arch Surg 2020, 405(6):767-776.

5. Sudo K, Xiao L, Wadhwa R, Shiozaki H, Elimova E, Taketa T, Blum MA, Lee JH, Bhutani MS, Weston B et al: Importance of surveillance and success of salvage strategies after definitive chemoradiation in patients with esophageal cancer. Journal of clinical oncology : official journal of the American Society of Clinical Oncology 2014, 32(30):3400-3405.

6. Markar S, Gronnier C, Duhamel A, Pasquer A, Thereaux J, du Rieu MC, Lefevre JH, Turner K, Luc G, Mariette C: Salvage Surgery After Chemoradiotherapy in the Management of Esophageal Cancer: Is It a Viable Therapeutic Option? Journal of clinical oncology : official journal of the American Society of Clinical Oncology 2015, 33(33):3866-3873.

7. Cohen C, Tessier W, Gronnier C, Renaud F, Pasquer A, Thereaux J, Gagniere J, Meunier B, Collet D, Piessen G et al: Salvage Surgery for Esophageal Cancer: How to Improve Outcomes? Annals of surgical oncology 2018, 25(5):1277-1286.

8. Faiz Z, Dijksterhuis WPM, Burgerhof JGM, Muijs CT, Mul VEM, Wijnhoven BPL, Smit JK, Plukker JTM: A meta-analysis on salvage surgery as a potentially curative procedure in patients with isolated local recurrent or persistent esophageal cancer after chemoradiotherapy. European journal of surgical oncology : the journal of the European Society of Surgical Oncology and the British Association of Surgical Oncology 2019, 45(6):931-940.

9. D'Journo XB, Thomas PA: Current management of esophageal cancer. Journal of thoracic disease 2014, 6(2):S253-264.

10. Katano A, Yamashita H, Nakagawa K: Re-irradiation of locoregional esophageal cancer recurrence following definitive chemoradiotherapy: A report of 6 cases. Molecular and clinical oncology 2017, 7(4):681-686.

11. Wu P, Liu J, Sun X, Li X, Xing L, Yu J: Enhanced radiosensitizing by sodium glycididazole in a recurrent esophageal carcinoma tumor model. Oncotarget 2017, 8(38):63871-63880.

12. Fakhrian K, Gamisch N, Schuster T, Thamm R, Molls M, Geinitz H: Salvage radiotherapy in patients with recurrent esophageal carcinoma. Strahlentherapie und Onkologie : Organ der Deutschen Rontgengesellschaft [et al] 2012, 188(2):136-142.

13. Zhou ZG, Zhen CJ, Bai WW, Zhang P, Qiao XY, Liang JL, Gao XS, Wang SS: Salvage radiotherapy in patients with local recurrent esophageal cancer after radical radiochemotherapy. Radiation oncology (London, England) 2015, 10:54. 
14. Xu X, Wang Z, Jiang S, Shang Y, Wu Y: Evaluating the optimal re-irradiation dose for locally recurrent esophageal squamous cell carcinoma after definitive radiotherapy. Radiation oncology (London, England) 2019, 14(1):191.

15. Zhang W, Luo Y, Wang X, Han G, Wang P, Yuan W, Dai SB: Doseescalated radiotherapy improved survival for esophageal cancer patients with a clinical complete response after standard-dose radiotherapy with concurrent chemotherapy. Cancer Manag Res 2018, 10:2675-2682.

16. Kobayashi R, Yamashita H, Okuma K, Shiraishi K, Ohtomo K, Nakagawa K: Salvage radiation therapy and chemoradiation therapy for postoperative locoregional recurrence of esophageal cancer. Diseases of the esophagus : official journal of the International Society for Diseases of the Esophagus 2014, 27(1):72-78.

17. Jingu K, Niibe Y, Yamashita H, Katsui K, Matsumoto T, Nishina T, Terahara A: Re-irradiation for oligorecurrence from esophageal cancer with radiotherapy history: a multi-institutional study. Radiation oncology (London, England) 2017, 12(1):146.

18. Ishihara R, Yamamoto S, lishi H, Takeuchi Y, Sugimoto N, Higashino K, Uedo N, Tatsuta M, Yano M, Imai A et al: Factors predictive of tumor recurrence and survival after initial complete response of esophageal squamous cell carcinoma to definitive chemoradiotherapy. International journal of radiation oncology, biology, physics 2010, 76(1):123-129.

19. Amini A, Ajani J, Komaki R, Allen PK, Minsky BD, Blum M, Xiao L, Suzuki A, Hofstetter W, Swisher S et al: Factors associated with local-regional failure after definitive chemoradiation for locally advanced esophageal cancer. Annals of surgical oncology 2014, 21(1):306-314.

20. Kim HW, Kim JH, Lee IJ, Kim JW, Lee YC, Lee CG, Park JJ, Youn YH, Park H: Local control may be the key in improving treatment outcomes of esophageal squamous cell carcinoma undergoing concurrent chemoradiation. Digestion 2014, 90(4):254-260.

21. Sun X, Wang L, Wang Y, Kang J, Jiang W, Men Y, Hui Z: High vs. Low Radiation Dose of Concurrent Chemoradiotherapy for Esophageal Carcinoma With Modern Radiotherapy Techniques: A MetaAnalysis. Front Oncol 2020, 10:1222.

22. Xiao L, Czito BG, Pang Q, Hui Z, Jing S, Shan B, Wang J: Do Higher Radiation Doses with Concurrent Chemotherapy in the Definitive Treatment of Esophageal Cancer Improve Outcomes? A MetaAnalysis and Systematic Review. Journal of Cancer 2020, 11(15):4605-4613.

23. Shridhar R, Almhanna K, Meredith KL, Biagioli MC, Chuong MD, Cruz A, Hoffe SE: Radiation therapy and esophageal cancer. Cancer Control 2013, 20(2):97-110.

24. Werner-Wasik M, Pequignot E, Leeper D, Hauck W, Curran W: Predictors of severe esophagitis include use of concurrent chemotherapy, but not the length of irradiated esophagus: a multivariate analysis of patients with lung cancer treated with nonoperative therapy. International journal of radiation oncology, biology, physics 2000, 48(3):689-696.

25. Li CC, Chen CY, Chien CR: Comparison of intensity-modulated radiotherapy vs 3-dimensional conformal radiotherapy for patients with non-metastatic esophageal squamous cell carcinoma 
receiving definitive concurrent chemoradiotherapy: A population-based propensity-score-matched analysis. Medicine (Baltimore) 2018, 97(22):e10928.

\section{Tables}

Table 1 Patients' characteristics $(n=125)$ 


\begin{tabular}{|c|c|c|c|c|}
\hline Characteristics & LD group $\otimes \square$ & HD group $₫ \% \rrbracket$ & $c^{2} / t$ & $P$ \\
\hline \multicolumn{5}{|l|}{ Gender } \\
\hline Male & $35 \rrbracket 60.3 \rrbracket$ & $49 \rrbracket 73.1 \rrbracket$ & 2.307 & 0.129 \\
\hline Female & $23 \rrbracket 39.7 \rrbracket$ & $18 \llbracket 26.9 \rrbracket$ & & \\
\hline \multicolumn{5}{|l|}{ Age } \\
\hline Mean \pm SD & $69.34 \pm 8.13$ & $68.18 \pm 7.53$ & 0.832 & 0.407 \\
\hline \multicolumn{5}{|l|}{ ECOG } \\
\hline $0-1$ & $48 \rrbracket 82.8 \rrbracket$ & $54 \llbracket 80.6 \rrbracket$ & 0.097 & 0.756 \\
\hline 2 & 10冈17.2】 & $13 \rrbracket 19.4 \rrbracket$ & & \\
\hline \multicolumn{5}{|c|}{ Recurrence pattern } \\
\hline PR & $35 \rrbracket 60.3 \rrbracket$ & $32 \bigotimes 47.8 \rrbracket$ & 1.614 & 0.446 \\
\hline LNR & 11ه19.0ष & $14 \llbracket 20.9 \rrbracket$ & & \\
\hline$P R+L N R$ & $12 \varangle 20.7 \rrbracket$ & $21 \rrbracket 31.3 \rrbracket$ & & \\
\hline \multicolumn{5}{|c|}{ Initial radiation dose (Gy) } \\
\hline Mean dose & $58.69 \pm 3.10$ & $59.22 \pm 2.76$ & 1.01 & 0.310 \\
\hline \multicolumn{5}{|c|}{ Time to recurrence (months) } \\
\hline$\leq 24$ & $36 \rrbracket 62.1 \rrbracket$ & $32 \rrbracket 47.8 \rrbracket$ & 2.57 & 0.109 \\
\hline$\bigotimes 24$ & $22 \bowtie 37.9 \rrbracket$ & $35 \rrbracket 52.2 \rrbracket$ & & \\
\hline \multicolumn{5}{|c|}{ Re-radiotherapy technology } \\
\hline 3D-CRT & $25 \rrbracket 43.1 \rrbracket$ & $34 \rrbracket 50.7 \rrbracket$ & 0.73 & 0.393 \\
\hline IMRT & $33 \rrbracket 56.9 \rrbracket$ & $33 \rrbracket 49.3 \rrbracket$ & & \\
\hline \multicolumn{5}{|l|}{ Chemotherapy } \\
\hline Yes & $25 \rrbracket 43.1 \rrbracket$ & $36 \rrbracket 53.7 \rrbracket$ & 1.405 & 0.236 \\
\hline No & $33 \rrbracket 56.9 \rrbracket$ & $31 \rrbracket 46.3 \rrbracket$ & & \\
\hline
\end{tabular}


Table 2: Toxic effects in the two groups (n)

\begin{tabular}{|lllllllll|}
\hline Toxicity & \multicolumn{2}{l}{ LD group } & & \multicolumn{7}{l|}{ HD group } \\
& Grade & Grade & Grade & Grade & Grade & Grade & Grade & Grade \\
& 1 & 2 & 3 & 4 & 1 & 2 & 3 & 4 \\
\hline $\begin{array}{l}\text { Radiation-induced } \\
\text { esophagitis }\end{array}$ & 19 & 13 & 2 & 0 & 29 & 14 & 3 & 0 \\
\hline Hematologic toxicity & 23 & 11 & 6 & 0 & 12 & 19 & 8 & 0 \\
\hline $\begin{array}{l}\text { Gastrointestinal } \\
\text { reactions }\end{array}$ & 6 & 5 & 3 & 0 & 12 & 7 & 3 & 0 \\
\hline $\begin{array}{l}\text { Radiation-induced } \\
\text { pneumonitis }\end{array}$ & 6 & 0 & 0 & 0 & 7 & 1 & 0 & 0 \\
\hline $\begin{array}{l}\text { Tracheitis } \\
\text { Skin reaction }\end{array}$ & 2 & 0 & 0 & 0 & 1 & 1 & 0 & 0 \\
\hline $\begin{array}{l}\text { Bleeding } \\
\text { Esophageal }\end{array}$ & 0 & 0 & 2 & 1 & 0 & 0 & 0 & 1 \\
\hline perforation/fistula & 0 & 0 & 3 & 2 & 0 & 0 & 1 & 1 \\
\hline Esophageal stenosis & 0 & 0 & 2 & 0 & 0 & 0 & 2 & 0 \\
\hline
\end{tabular}

Table 3: Prognostic factors for patient survival 


\begin{tabular}{|c|c|c|c|c|c|c|c|}
\hline \multirow[t]{2}{*}{ Variables } & \multirow[t]{2}{*}{ Number } & \multicolumn{3}{|c|}{ Univariate } & \multicolumn{3}{|c|}{ Multivariate } \\
\hline & & HR & $95 \% \mathrm{Cl}$ & $P$ & HR & $95 \% \mathrm{Cl}$ & $P$ \\
\hline Gender & & 0.758 & $\begin{array}{l}0.505- \\
1.138\end{array}$ & 0.181 & & & \\
\hline Male & 84 & & & & & & \\
\hline Female & 41 & & & & & & \\
\hline Age (years) & & 1.519 & $\begin{array}{l}1.046- \\
2.208\end{array}$ & 0.028 & 1.472 & $\begin{array}{l}1.010- \\
2.144\end{array}$ & 0.044 \\
\hline$\otimes 70$ & 71 & & & & & & \\
\hline$\geq 70$ & 54 & & & & & & \\
\hline ECOG & & 1.593 & $\begin{array}{l}1.004- \\
2.527\end{array}$ & 0.048 & 1.641 & $\begin{array}{l}1.027- \\
2.621\end{array}$ & 0.038 \\
\hline $0-1$ & 102 & & & & & & \\
\hline 2 & 23 & & & & & & \\
\hline Recurrence pattern & & 1.149 & $\begin{array}{l}0.915- \\
1.444\end{array}$ & 0.232 & & & \\
\hline PR & 67 & & & & & & \\
\hline LNR & 25 & & & & & & \\
\hline$P R+L N R$ & 33 & & & & & & \\
\hline $\begin{array}{l}\text { Time to recurrence } \\
\text { (months) }\end{array}$ & & 0.507 & $\begin{array}{l}0.346- \\
0.745\end{array}$ & 0.001 & 0.484 & $\begin{array}{l}0.328- \\
0.713\end{array}$ & $<0.001$ \\
\hline$\leq 24$ & 68 & & & & & & \\
\hline$\bigotimes 24$ & 57 & & & & & & \\
\hline Chemotherapy & & 0.758 & $\begin{array}{l}0.526- \\
1.094\end{array}$ & 0.139 & & & \\
\hline Yes & 61 & & & & & & \\
\hline No & 64 & & & & & & \\
\hline Re-irradiation dose & & 0.626 & $\begin{array}{l}0.432- \\
0.908\end{array}$ & 0.013 & 0.638 & $\begin{array}{l}0.439- \\
0.927\end{array}$ & 0.018 \\
\hline 50-54Gy & 58 & & & & & & \\
\hline $55-60 G y$ & 67 & & & & & & \\
\hline
\end{tabular}


Figures

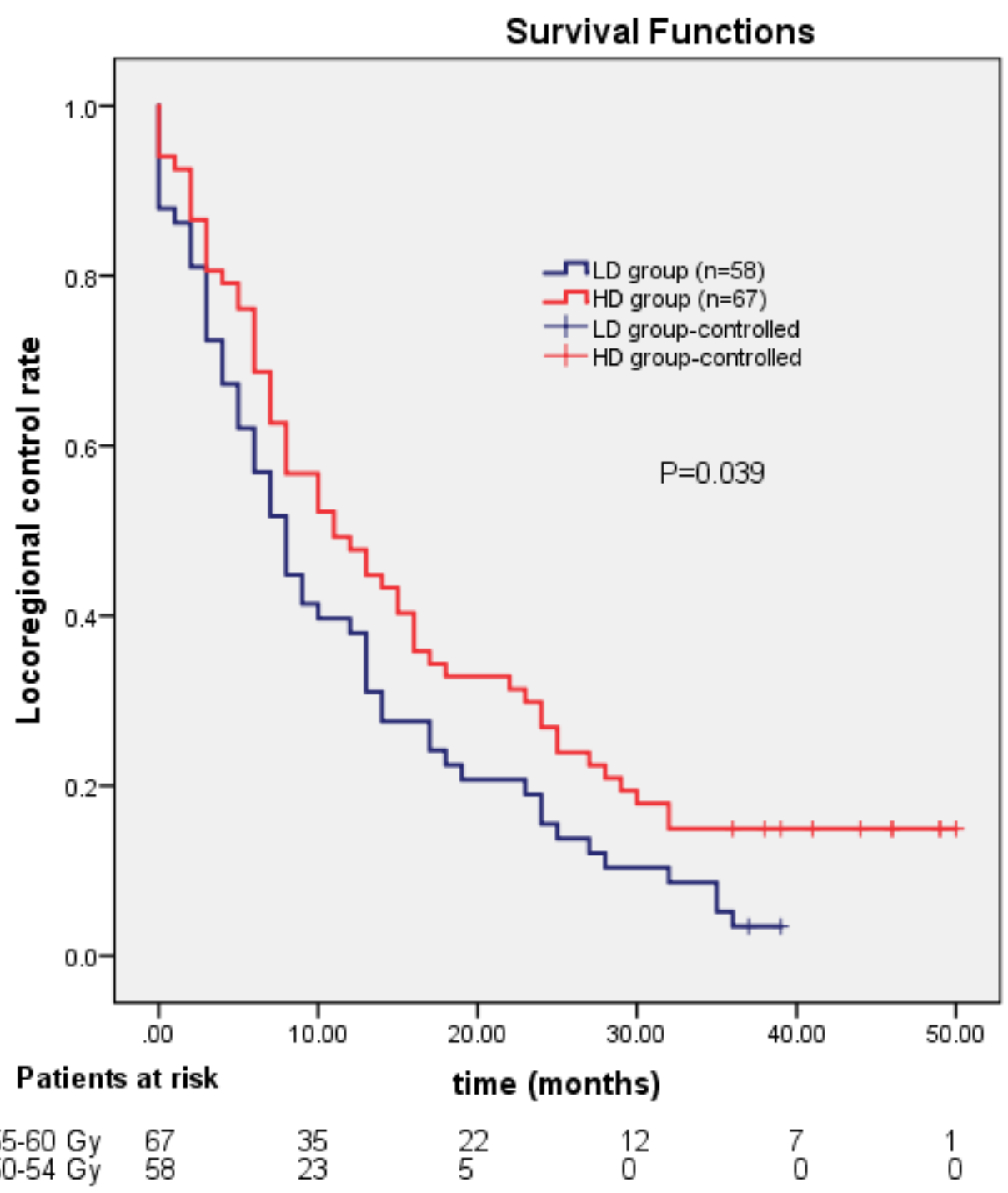

Figure 1

There was a significant difference between locoregional control rate in the LD group and HD group. 


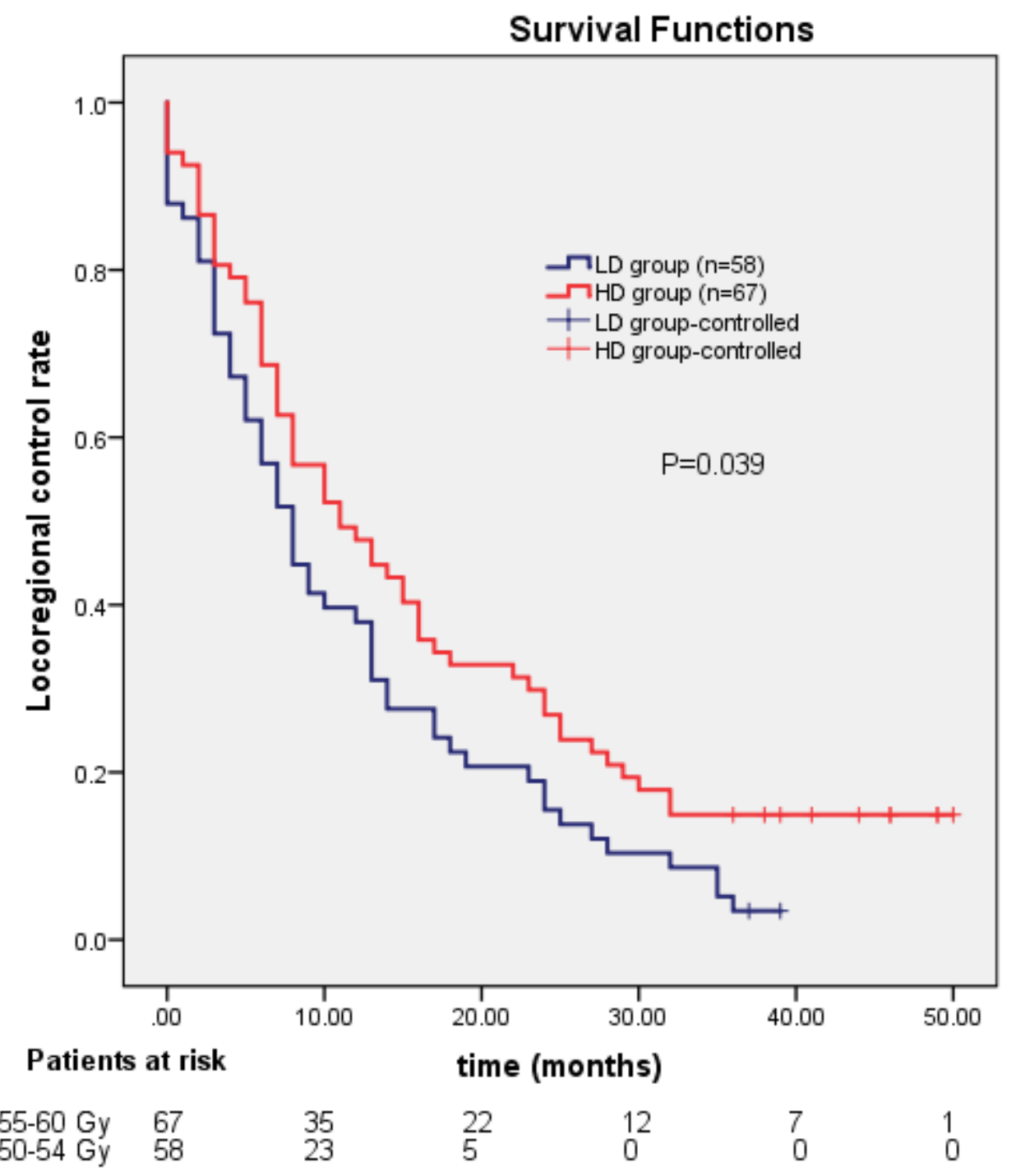

\section{Figure 1}

There was a significant difference between locoregional control rate in the LD group and HD group. 


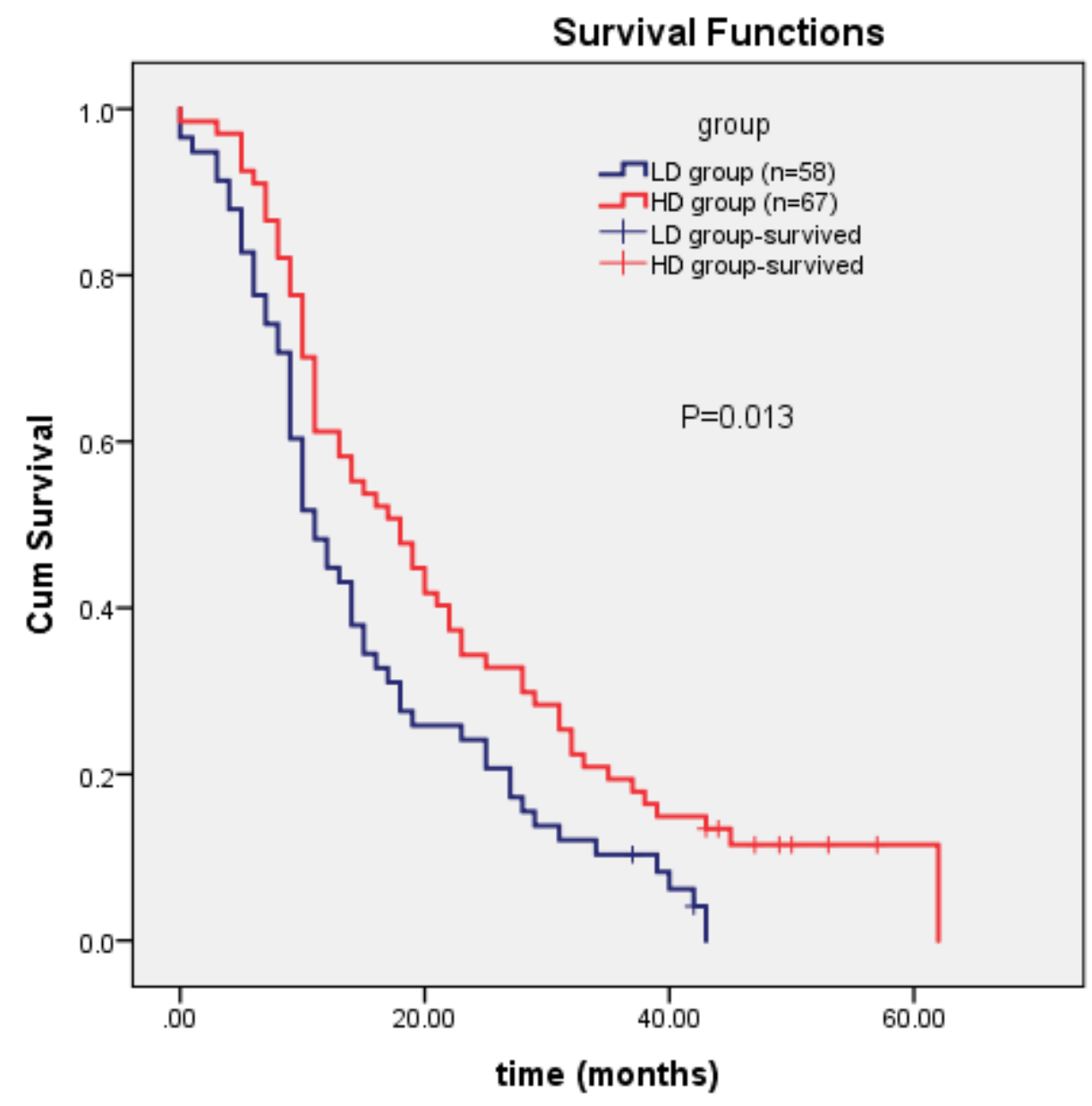

Figure 2

Survival curve of patients in the LD and HD groups after re-irradiation therapy. 


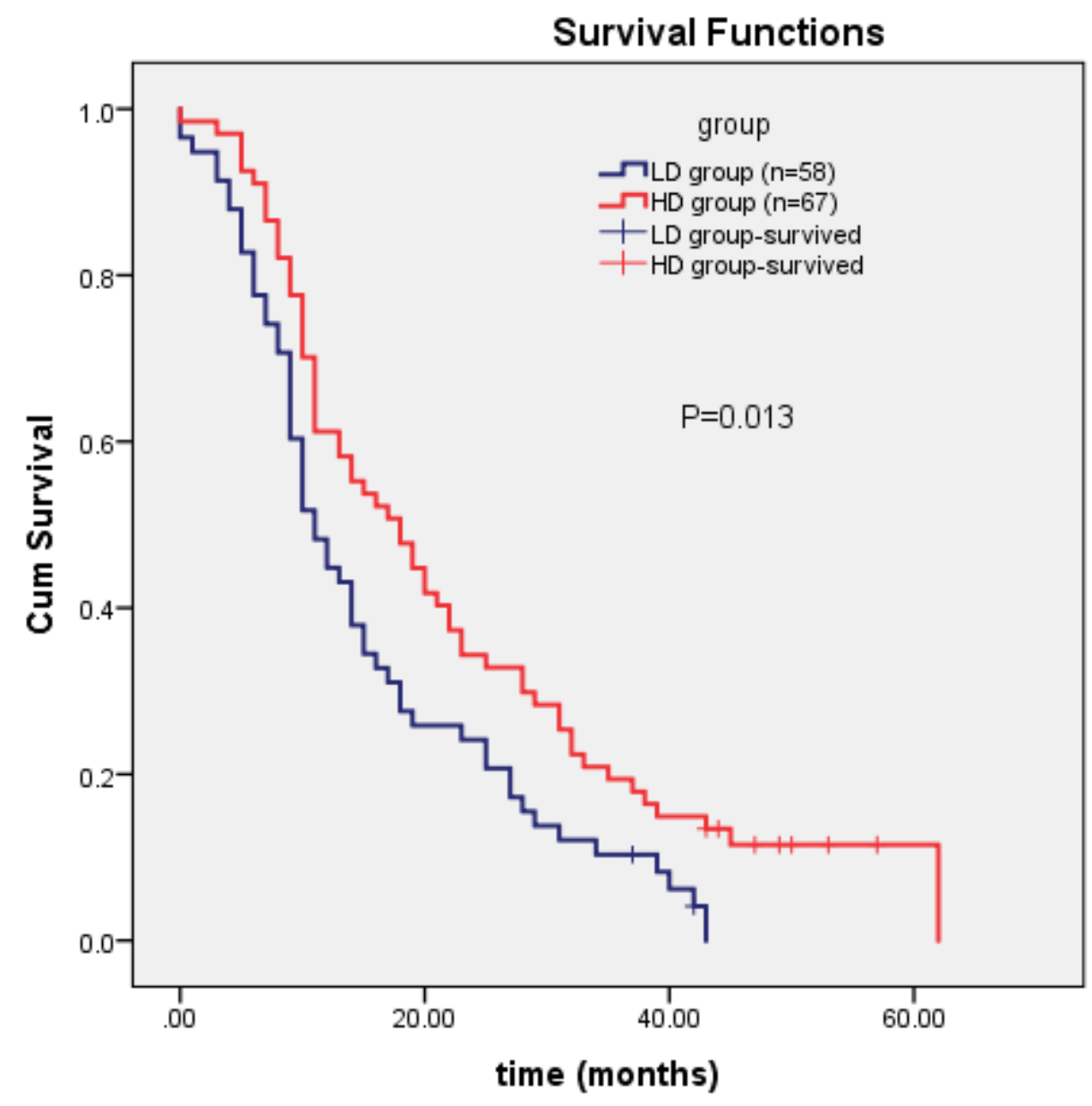

Figure 2

Survival curve of patients in the LD and HD groups after re-irradiation therapy. 

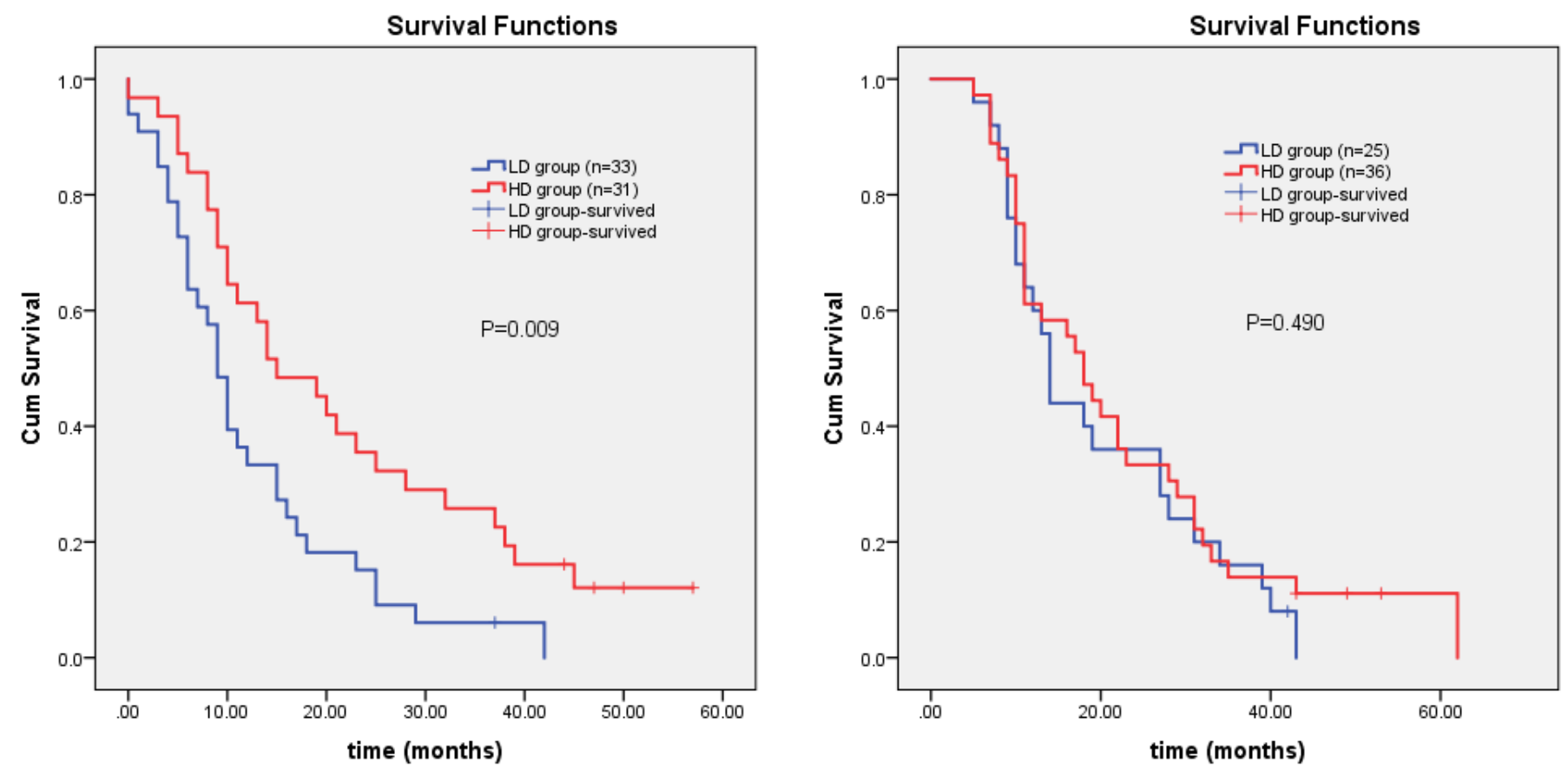

Figure 3

Patients were stratified according to whether they accepted chemotherapy. (A) Survival curve of patients in the LD or HD group after treatment with re-irradiotherapy alone. Patients in the HD group had a significantly better median survival time compared with patients in the LD group (15 months vs 9 months, $P=0.009$ ). (B) Survival curve of patients in the LD and HD group with chemoradiotherapy. The median survival time of patients receiving chemoradiotherapy was 18 months in the HD group and 14 months in the LD group $(P=0.490)$.
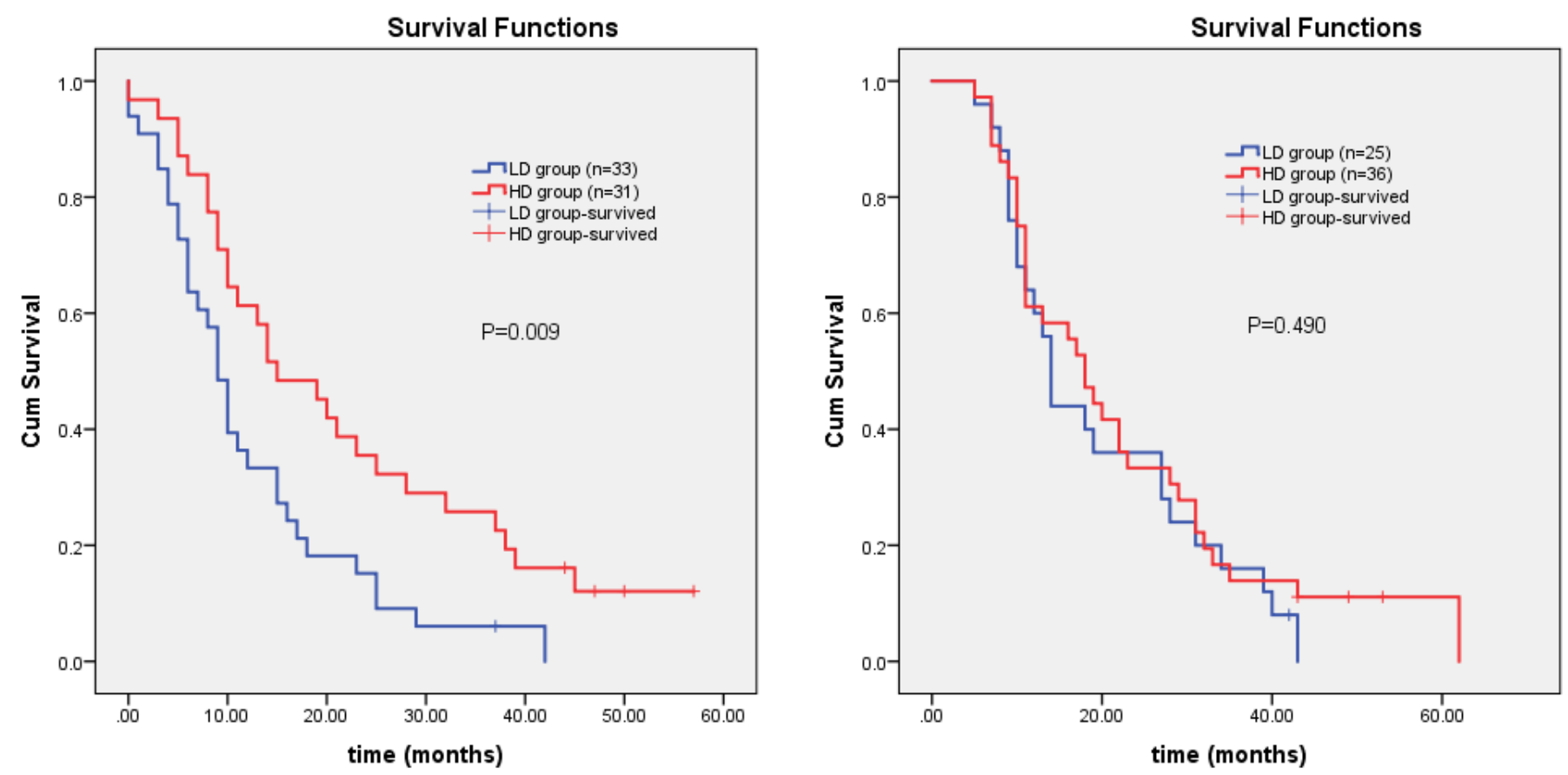


\section{Figure 3}

Patients were stratified according to whether they accepted chemotherapy. (A) Survival curve of patients in the LD or HD group after treatment with re-irradiotherapy alone. Patients in the HD group had a significantly better median survival time compared with patients in the LD group ( 15 months vs 9 months, $P=0.009$ ). (B) Survival curve of patients in the LD and HD group with chemoradiotherapy. The median survival time of patients receiving chemoradiotherapy was 18 months in the HD group and 14 months in the LD group $(P=0.490)$.
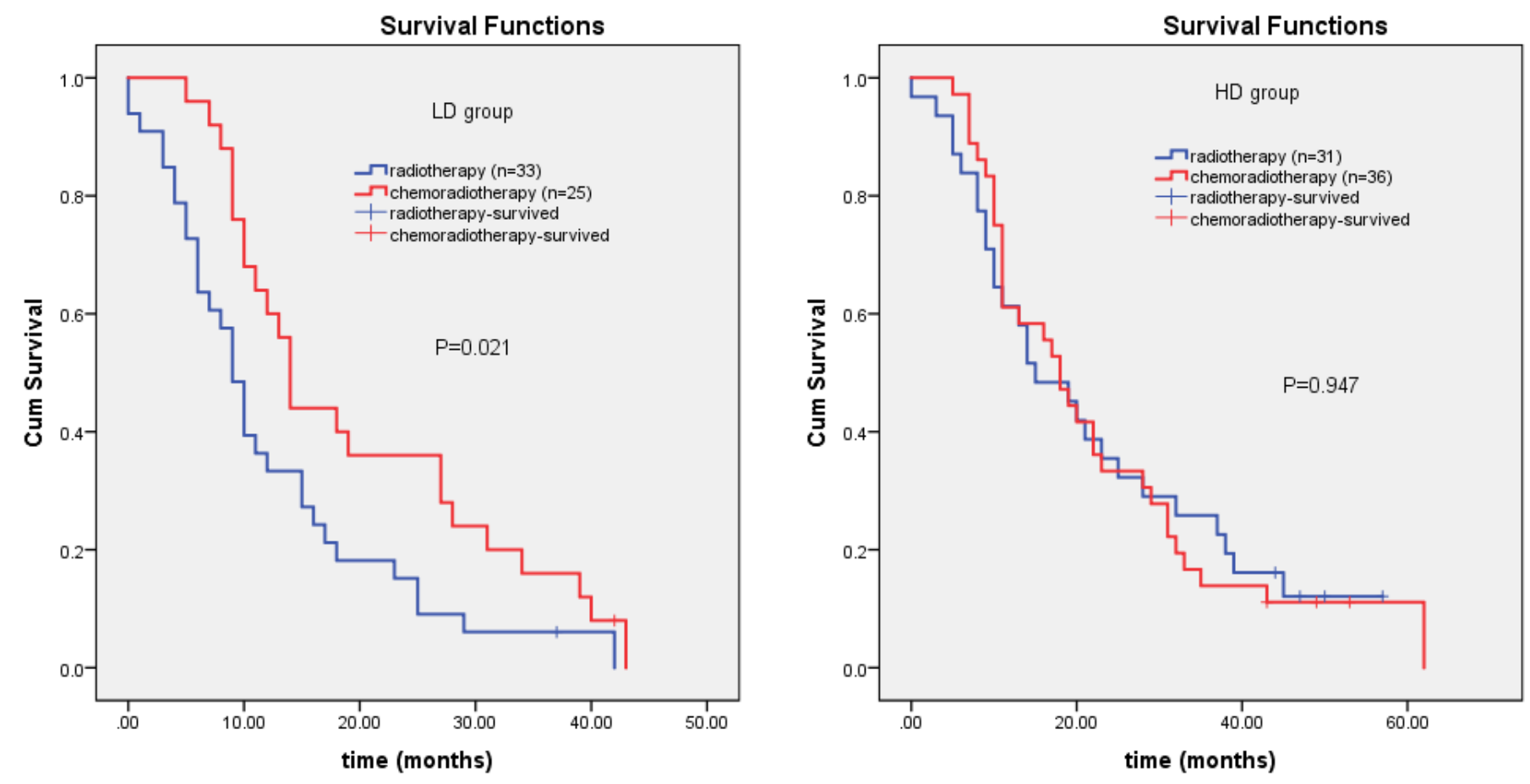

\section{Figure 4}

Survival curve based on the patients who received chemotherapy in each group. (A) Patients in the LD group with chemoradiotherapy showed superior median survival time than patients who received reirradiotherapy alone (14 months vs 9 months, $P=0.021)$. (B) Patients with chemotherapy did not show better median survival time compared to those without chemotherapy (18 months vs 15 months) in the HD group $(P=0.947)$. 

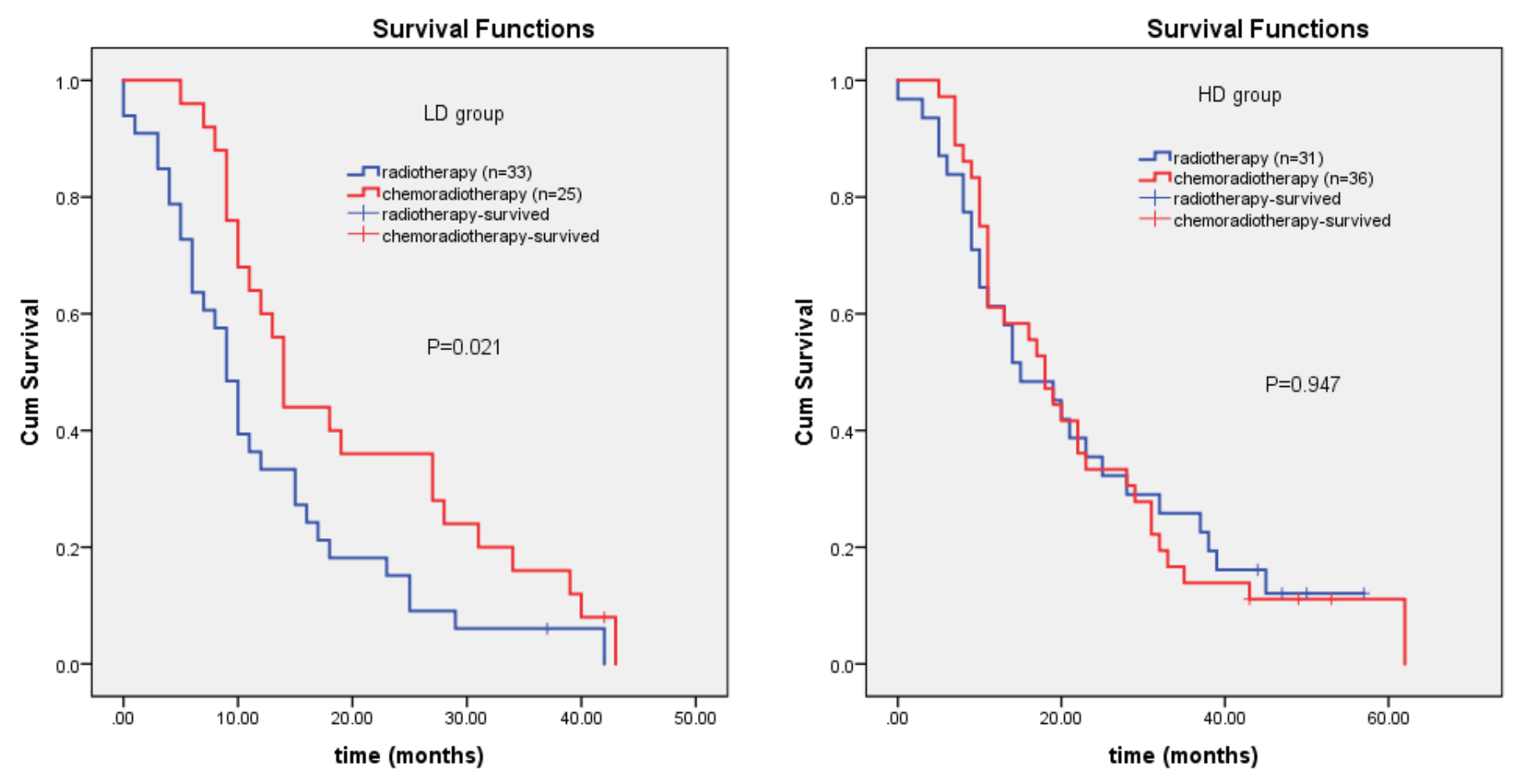

\section{Figure 4}

Survival curve based on the patients who received chemotherapy in each group. (A) Patients in the LD group with chemoradiotherapy showed superior median survival time than patients who received reirradiotherapy alone (14 months vs 9 months, $P=0.021)$. (B) Patients with chemotherapy did not show better median survival time compared to those without chemotherapy (18 months vs 15 months) in the HD group $(P=0.947)$. 